\title{
The Importance of Measurement for Quality Improvement: Submaximal Cardiac Stress Testing
}

\author{
Mary Beth Farrell, CNMT, FSNMMI-TS \\ Intersocietal Accreditation Commission, Ellicott City, Maryland
}

CE credit: For CE credit, you can access the test for this article, as well as additional JNMT CE tests, online at https://www.snmmilearningcenter.org. Complete the test online no later than June 2023. Your online test will be scored immediately. You may make 3 attempts to pass the test and must answer $80 \%$ of the questions correctly to receive $1.0 \mathrm{CEH}$ (Continuing Education Hour) credit. SNMMI members will have their CEH credit added to their VOICE transcript automatically; nonmembers will be able to print out a CE certificate upon successfully completing the test. The online test is free to SNMMI members; nonmembers must pay $\$ 15.00$ by credit card when logging onto the website to take the test.

\begin{abstract}
One of the most effective techniques to improve quality is to measure quality. The aim of this article is to defend the importance of quality measurement in nuclear medicine using the longstanding problem of submaximal exercise stress testing. Numerous evidence-based guidelines and research studies establish the importance of maximal stress testing for myocardial perfusion imaging. The 3 cutoffs for adequate cardiac stress that must be met are expenditure of 5 metabolic equivalents of energy, production of symptoms (e.g., fatigue or shortness of breath), and attainment of $85 \%$ of the maximum predicted heart rate. Measurement and evaluation of these 3 criteria along with several other metrics can help to validate one aspect of laboratory quality related to myocardial perfusion imaging accuracy.
\end{abstract}

Key Words: exercise stress testing; submaximal stress; quality improvement; measurement; guideline adherence

J Nucl Med Technol 2020; 48:122-125

DOI: 10.2967/jnmt.120.244525

$\mathbf{T}$ he Institute of Medicine defines quality as "the degree to which health services for individuals and populations increase the likelihood of desired health outcomes and are consistent with current professional knowledge" (1). Key to this definition is a comprehensive understanding of 2 phrases: "health outcomes" and "consistent with professional knowledge." Health outcomes, otherwise referred to as patient outcomes, as defined by the World Health Organization are "a change in the health of an individual, group of people, or population that is attributable to an intervention or series of interventions" (2). Professional knowledge, otherwise referred to as evidence-based medicine, is the careful, precise, judicious, and sensible use of

Received Mar. 3, 2020; revision accepted Mar. 30, 2020.

For correspondence or reprints contact: Mary Beth Farrell, P.O. Box 324, Great Cacapon, WV 25422.

E-mail: marybethfarrell2016@gmail.com

Published online Apr. 10, 2020.

COPYRIGHT (C) 2020 by the Society of Nuclear Medicine and Molecular Imaging. current knowledge and evidence when making decisions on the care of a patient (3). It is important to note that evidence-based medicine values use of the soundest information available and skillfully combines a physician's clinical experience and expertise with a patient's desires.

One of the most effective ways to improve health outcomes is through the application of professional, evidencebased guidelines (4). Evidence-based imaging guidelines provide concise instructions on how to perform a procedure, thus ensuring standardization and improving quality (5). However, the question arises, "How do you know you are improving quality?" One way to assess quality and to safeguard improvement is through measurement. Laboratories must select appropriate metrics and apply the correct knowledge base to improve quality.

The aim of this article is to defend the importance of measurement in improving quality. This article will describe a long-standing quality issue in nuclear cardiology that is recently getting renewed attention: submaximal stress testing. The paper will describe that problem, explain the evidencebased guidelines related to the problem, and suggest metrics to quantify and correct the problem.

\section{IMPORTANCE OF MEASURING QUALITY}

There is a plethora of axioms and reasons to support the importance of measurement in improving quality. Peter Drucker, a pioneer in quality, is credited with stating, "you can't manage what you can't measure" (6). By this, Drucker meant you cannot determine whether you are successful unless you first define success and track it. When success is bound by a metric, progress can be quantified, and actions can be adjusted to produce the desired outcome. A quote from H. James Harrington, another guru of quality, is more prescriptive than Drucker. Harrington is credited with stating, "Measurement is the first step that leads to control and eventually to improvement. If you can't measure something, you can't understand it. If you can't understand it, you can't control it. If you can't control it, you can't improve it" (7). 
One practical reason for measuring is that it increases confidence in decision-making (8). For example, baseball coaches use players' batting averages to determine where to put them in the line-up. Measurement helps an individual know what is going on, or the status of things. Teachers use the results of tests to assess how well a student comprehends the material. Measurement monitors, or keeps tabs, and is an indicator of change-whether that change is an improvement or a deterioration. If decline is noted, measurements highlight the need for improvement. Metrics provide a framework to keep people focused on what is most important. It allows them to set goals and then evaluate progress.

Through measurement, a view of the entire system can be obtained, providing a more comprehensive understanding of system function as opposed to anecdotal occurrences. Systematic assessments as opposed to haphazard, random efforts to evaluate and enhance quality produce better longterm results (9). Deliberate data collection and analyses produce more consequential system transformation than do knee-jerk reactions and policing actions. By evaluating systems, long-term "cures" are obtainable as opposed to just treating the system's "symptoms."

Perhaps the most definitive reason for measuring quality in health care was provided by the Agency for Healthcare Research and Quality. In its 2012 National Healthcare Quality Report, it noted that "patients receive the proper diagnosis and care only about 55\% of the time" (10). Although this figure represents the continuum of patients and diagnoses, it is appropriate for diagnostic medicine because clinicians derive most diagnoses based on the results of an imaging procedure or clinical laboratory test.

\section{QUALITY IN ADVANCED DIAGNOSTIC IMAGING}

Hendee et al. estimated that between $20 \%$ and $50 \%$ of advanced diagnostic imaging tests (i.e., nuclear medicine, CT, and MRI) do not provide results improving patient outcomes (11). There are many reasons why a diagnostic imaging test may fail to provide meaningful information. For example, the referring physician may not have ordered the correct scan to answer the clinical question. Referring physicians must order the most appropriate test from the profusion of available imaging options, with new tests and clinical indications continually emerging. Suboptimal imaging findings can occur if the patient was not adequately prepared for the test or the test is performed incorrectly. For example, if a patient does not fast before a cardiac PET scan, the heart will fail to accumulate the radiotracer and the images will be of poor quality (12).

One diagnostic accuracy problem in nuclear cardiology has received recent public attention. A study published in JACC: Cardiovascular Imaging and picked up by the media in April 2019 found considerable variation in the performance of stress testing at U.S. Department of Veterans Affairs hospitals (13). At issue was the diagnostic accuracy of myocardial perfusion imaging due to submaximal cardiac stress testing.

\section{SUBMAXIMAL STRESS TESTING AND EVIDENCE-BASED GUIDELINES}

The goal of stress myocardial perfusion imaging is to provoke myocardial ischemia (decreased blood flow) to a region of the heart at risk of infarction. In essence, the objective is to diagnose coronary artery disease by comparing the blood flow to the heart muscle during stress and resting conditions using radioactive tracers and nuclear medicine scanners (14). For the test to be accurate, patients must adequately exert themselves during an exercise treadmill test to produce ischemia. If a patient cannot exercise to sufficient levels, the test can be performed using pharmacologic agents to simulate exercise. The problem is that in many nuclear cardiology stress laboratories, the radioactive tracer is being injected too soon or before the patient has reached the ischemic threshold. Bluntly stated, many facilities inject the moment the patient reaches $85 \%$ of the maximum predicted heart rate. The result is a false-negative test-meaning the imaging results do not show ischemia, incorrectly indicating the patient's coronary arteries are healthy when in fact they may contain a blockage. Without an adequate cardiac stress test, there is no way to determine whether the patient is or is not at risk of infarction.

A substantial number of clinical research studies have evaluated and determined an adequate level of exercise in most adults to produce ischemia. There are 3 cutoffs for adequate cardiac stress that the patient must meet: the patient must expend 5 metabolic equivalents (METs) of energy (a workload of about 6 min using the standard Bruce protocol for the treadmill), indicating moderate exercise sufficient to provoke ischemia; the heart rate must reach a level higher than $85 \%$ of the maximum predicted heart rate based on age (maximum predicted heart rate $=220-$ age); and the patient must have symptoms such as fatigue, shortness of breath, chest pain, or electrocardiogram changes indicative of ischemia $(15,16)$. Basically, the patient must be markedly fatigued. The published evidence-based, clinical guidelines from various professional imaging societies mandate that the patient must meet these 3 exercise requirements or undergo a pharmacologic stress test.

\section{SUBMAXIMAL STRESS QUALITY IMPROVEMENT}

To assess current laboratory adherence to the evidencebased guidelines for stress myocardial perfusion imaging and set baseline levels, the following metrics are suggested: patient identification number, sex, age, maximum predicted heart rate, exercise protocol, METs achieved, total exercise time, peak heart rate, peak systolic blood pressure, ratepressure product, symptoms, electrocardiogram findings, and myocardial perfusion imaging findings and whether the patient performed maximal-stress exertion or required a pharmacologic stress test (Fig. 1).

\section{Metrics Rationale}

The most critical of these metrics are the peak heart rate achieved, the METs achieved, whether the patient had 


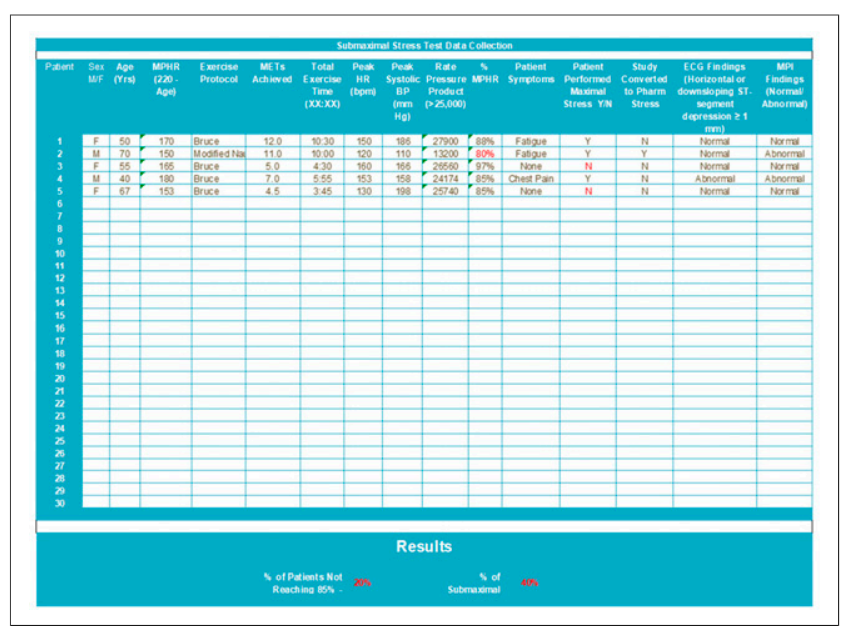

FIGURE 1. Sample data collection form with critical metrics to ascertain adequacy of cardiac stress testing before myocardial perfusion imaging. The most critical variables are percentage of maximum predicted heart rate, METs achieved, and patient symptoms. BP = blood pressure; ECG = electrocardiography; $\mathrm{HR}=$ heart rate; MPHR = maximum predicted heart rate; $\mathrm{MPI}=$ myocardial perfusion imaging.

symptoms, whether the patient was markedly fatigued (maximal stress), and the exercise time. These 5 metrics are essential. The other metrics provide a quality check. The reason these metrics are needed is that a patient could exercise less than 2 min and, because of severe deconditioning or obesity, achieve greater than $85 \%$ of the maximum predicted heart rate. The imaging would result in a false-negative result in this situation because less than 2 min of exercise are not sufficient to provoke maximal coronary artery dilation. The METs achieved and the heart rate are also important metrics, considered together, because an athlete could exercise for $6 \mathrm{~min}$ and achieve 5 METs, but because of conditioning, the patient's heart rate may be significantly less than $85 \%$ of the maximum predicted value, and thus the coronary arteries may not be maximally dilated. Similarly, a patient could walk less than 2 min, exert less than 5 METs, and have crushing chest pain but still not have maximal coronary artery dilation. The concern with this scenario is that the chest pain could indicate coronary artery disease, but because the patient did not exercise long enough, the image findings may not be abnormal.

Several of the other metrics are germane to improving quality, the first being whether the patient was converted to a pharmacologic stress test if the 3 conditions were not met. If the 3 conditions are not met, there is an increased likelihood of false-negative results, meaning that patients are told they are disease-free when, in fact, they may have blockages in their coronary arteries increasing the likelihood of future myocardial infarction. Relatedly, another important quality metric is whether the scan results were normal or abnormal. If the laboratory truly wants to improve quality, it should also compare the imaging results with a gold standard such as cardiac catheterization (17). Comparison to a gold standard, or correlation, is a measure of the number of false-positives, false-negatives, true-positives, and true-negatives or, in other words, the accuracy of the test.

\section{Metrics Goals}

There are 2 goals associated with the collection of data from these metrics. First, a laboratory can determine the level of accuracy associated with myocardial perfusion imaging. As mentioned previously, 20\%-50\% of patients undergoing advanced diagnostic imaging do not receive results or information improving outcomes. Only through accurate results can we hope to improve patient outcomes. One way to improve the accuracy of a test is through proper performance. Thus, adherence to evidence-based procedure guidelines is a method for enhancing performance. The second goal of these metrics is to establish the degree of laboratory adherence to the clinical guidelines.

\section{CONCLUSION}

The Institute of Medicine's definition of quality emphasizes patient outcomes through the application of evidence-based knowledge. There are several evidence-based imaging guidelines for the performance of stress myocardial perfusion imaging. These guidelines provide concise instructions on how to perform the test to obtain the highest level of accuracy. However, a laboratory cannot just assume tests are being performed correctly, nor can it assume tests comply with the guidelines. The only way to ensure these things, and thereby improve quality, is through measurement.

This paper provided a rationale for measuring the quality of cardiac exercise stress testing associated with myocardial perfusion imaging. At issue is the performance of maximal exercise to achieve accurate imaging results. Three conditions must be met to substantiate a maximal exercise test. The patient must exercise to a level of 5 METs or approximately $6 \mathrm{~min}$ on the treadmill. The patient's heart rate must reach higher than $85 \%$ of the maximum predicted heart rate, and the patient must be symptomatic. These 3 conditions have been suggested, along with several other exercise attributes, as practical metrics to ascertain quality. From the measurement of these metrics, adherence to evidence-based guidelines and the accuracy of the cardiac stress test for myocardial perfusion imaging can be demonstrated. Affirming the words of H. James Harrington, measurement of exercise stress testing adequacy furthers understanding. Understanding the current performance of stress testing can assist a laboratory in controlling performance, and by controlling performance, a laboratory can facilitate improvement.

\section{DISCLOSURE}

Mary Beth Farrell is an employee of the Intersocietal Accreditation Commission. No other potential conflict of interest relevant to this article was reported. 


\section{REFERENCES}

1. Institute of Medicine (IOM). Crossing the Quality Chasm: A New Health System for the 21st Century. Washington, DC: National Academy Press; 2001.

2. Definitions: emergencies. World Health Organization website. https://www.who.int/ hac/about/definitions/en/. Accessed April 14, 2020.

3. Masic I, Miokovic M, Muhamedagic B. Evidence based medicine: new approaches and challenges. Acta Inform Med. 2008;16:219-225.

4. Grimshaw JM, Russell IT. Effect of clinical guidelines on medical practice: a systematic review of rigorous evaluations. Lancet. 1993;342:1317-1322.

5. Graham ID, Harrison MB. Evaluation and adaptation of clinical practice guidelines. Evid Based Nurs. 2005;8:68-72.

6. Prusak L. What can't be measured. Harvard Business Review website. https:// hbr.org/2010/10/what-cant-be-measured. Published October 7, 2010. Accessed April 14, 2020.

7. Hutt M, Speh T. Business Marketing Management. Mason, OH: South-Western Cengage Learning; 2009.

8. Ogrinc GS, Headrick LA, Moore SM, Barton AJ, Dolansky MA, Madigosky WS. Fundamentals of Health Care Improvement: A Guide to Improving your Patients' Care. 2nd ed. Oakbrook Terrace, IL: Joint Commission Resources; 2012.

9. Farrell MB, Abreu SH. A practical guide to quality improvement in nuclear medicine. J Nucl Med Technol. 2012;40:211-219.
10. 2012 national healthcare quality report. U.S. Department of Health and Human Services website. https://archive.ahrq.gov/research/findings/nhqrdr/nhqr12/index.html. Last reviewed June 2013. Accessed April 14, 2020.

11. Hendee WR, Becker GJ, Borgstede JP, et al. Addressing overutilization in medical imaging. Radiology. 2010;257:240-245.

12. Dilsizian V, Bacharach SL, Beanlands RS, et al. ASNC imaging guidelines/ SNMMI procedure standard for positron emission tomography (PET) nuclear cardiology procedures. J Nucl Cardiol. 2016;23:1187-1226.

13. Shah NR, Ahmed S, Winchester D, et al. Facility-level variation in stress test utilization in veterans with ischemic heart disease. JACC Cardiovasc Imaging. 2019;12:1292-1293.

14. Pagnanelli RA, Camposano H. Pharmacologic stress testing with myocardial perfusion imaging. J Nucl Med Technol. 2017;45:249-252.

15. Henzlova M, Duvall L, Einstein A, Travin M, Verberne H. ASNC imaging guidelines for SPECT nuclear cardiology procedures: stress, protocols, and tracers. J Nucl Cardiol. 2016;23:606-639.

16. Sharif S, Always S. The diagnostic value of exercise stress testing for cardiovascular disease is more than just ST segment changes: a review. J Integr Cardiol. 2016;2:341-355.

17. Koller D. Assessing diagnostic performance in nuclear cardiology. J Nucl Cardiol. 2002;9:114-123. 